\title{
An association study of Taq 1 A ANKK1 and C957T and - 141C DRD2 polymorphisms in adults with internet gaming disorder: a pilot study
}

Soo-Hyun Paik, Mi Ran Choi, Su Min Kwak, Sol Hee Bang, Ji-Won Chun, Jin-Young Kim, Jihye Choi, Hyun Cho, Jo-Eun Jeong and Dai-Jin Kim*

\begin{abstract}
Background: Though Internet gaming disorder (IGD) is considered to share similar genetic vulnerability with substance addictions, little has been explored about the role of the genetic variants on IGD. This pilot study was designed to investigate the association of the Taq $1 A$ polymorphism of the ankyrin repeat and kinase domain containing 1 (ANKK1) gene and C957T and - 141C of the dopamine D2 receptor (DRD2) with IGD and their role on the personality and temperament traits in IGD among adult population.

Methods: Sixty-three subjects with IGD and 87 control subjects who regularly played Internet games were recruited. Self-administered questionnaires on self-control, dysfunctional impulsivity, and temperament and character domains were done. The Taq1A ANKK1 and the C957T and - 141C ins/del from the DRD2 genes were genotyped using the specific TaqMan PCR assay.

Results: The distributions of allele and genotype frequencies were not significantly different between the IGD and control groups in both genders. In male, excessive gaming and use of gaming to escape from a negative feeling were associated with the del-genotype of the - 141C. Among IGD, the del+ genotype was associated with higher novelty seeking. Logistic regression showed no predictive value of these polymorphisms for IGD when using age and gender as covariates.
\end{abstract}

Conclusions: Though no direct association of the Taq1A ANKK1 and C957T DRD2 variants with IGD were observed, the $-141 C$ polymorphism may play a role in IGD via mediating symptoms or temperament traits.

Keywords: Internet gaming disorder, Dopamine D2 receptor, ANKK1, TaqMan assay, Personality and temperament

\section{Introduction}

Internet gaming disorder (IGD) is conceptualized as a behavioral addiction that excessive preoccupation and loss of control over Internet gaming eventually leads to the functional impairment [1]. Growing evidence suggested that IGD resembles substance additions in phenomenology, genetic and environmental risk factors, and

\footnotetext{
*Correspondence: kdj922@catholic.ac.kr

Department of Psychiatry, Seoul St. Mary's Hospital, The Catholic

University of Korea College of Medicine, Seoul, 222, Banpo-daero,

Seocho-gu, Seoul 06591, South Korea
}

neurobiological mechanisms [2]. Individuals with IGD showed impulsivity and response disinhibition in $\mathrm{Go} /$ No-Go tasks [3], tendency to make risky decision in Cups tasks [4], and increased craving when gaming-related cues were presented [5]. Furthermore, dysregulation of the dopamine D2 receptors in the striatum was observed in Internet addiction [6]. On account of this, a previous study revealed the association of the Taq1A polymorphism of the ankyrin repeat and kinase domain containing 1 (ANKK1) gene, which was associated with altered availability of dopamine D2 receptor, with IGD in male adolescents [7]. Family and twin studies have shown that 
the genetic factors accounted for $41-66 \%$ of the variance of the Internet addiction [8-10], which was comparable to that of substance addictions [11]. Accordingly, the identification of genetic vulnerabilities is considered as providing better understanding of the neurobiology of IGD and improving intervention outcomes. However, little has been explored on the role of the genetic variants on IGD.

The genetic polymorphisms that potentially alter the availability and expression of the dopamine D2 receptor have been known to be associated with both substance and behavioral addictions $[12,13]$. The Taq1A polymorphism (SNP ID: rs1800497), which is located in exon 8 of $A N K K 1$, adjacent to the terminal codon of the DRD2 (OMIM*126450; gene map locus 11q23.2), is known to alter binding specificity and reduce DRD2 expression in striatum and associated structures. A significant number of genetic studies have found the association of the Taq1A polymorphism with substance addiction and impulsivity and psychopathic traits in alcohol dependence [14-16]. The C957T polymorphism (SNP ID: rs1799732), which is located on exon 7 of the DRD2 (gene map locus 11q23), is known to alter DRD2 availability and be associated with alcoholism with higher prevalence of the CC genotype $[17,18]$. The $-141 \mathrm{C}$ Ins/Del polymorphism (SNP ID: rs6277), which is located on promoter of the DRD2 (gene map locus 11q23), alters striatal D2 receptor binding potentials, and is associated with alcoholism $[16,19$, $20]$. Given the gene performance and the association with substance addiction, the three polymorphisms may play a crucial role in IGD. To date, however, no study has investigated the association of these polymorphisms with IGD.

Personality and temperament traits such as high neuroticism, psychoticism, sensational seeking, and reward dependence and low self-directedness and cooperativeness were associated with IGD [21-24]. Personality traits are strongly influenced by genetics. Twin studies which reported individual differences in personality showed about $50 \%$ of heritability, which means that about half of the variation in personality traits is affected by genetics $[25,26]$. Though this assumption is oversimplified and several studies performed in healthy volunteers have reported no association between the variants of DRD2 gene and personality and temperament traits $[27,28]$, relations were found in substance and behavioral addiction. For example, the Taq1A A1 carriers of pathological gambling had higher harm avoidance and lower self-directedness than non-A1 carriers, and the C957T T carriers of alcohol dependence had higher psychopathic tendency $[18,29]$. Given that genetics could influence addictive disorders either directly or mediated via personality traits [27], genetics may better account for the vulnerability for IGD when personality and character traits are taken into account. At present, two studies have investigated the association of genetic variants with temperament traits in excessive Internet users: the Taq1A polymorphism with higher reward dependence and the short allelic variant of the serotonin transporter gene (SS-5HTTLPR) with higher harm avoidance $[7,30]$. Despite, these studies only included male adolescents who normally manifested higher sensation seeking and risk taking behavior, used rather subjective definition of excessive Internet users, and represented only personality dimensions of interest. Accordingly, a need to investigate the genetics of IGD with consideration of the temperament and personality traits in adults, who have more stable personality structure than adolescents, is emerging.

The present pilot study was designed to investigate the association of the genetic polymorphisms of $D R D 2$ (-141C and C957T) and ANKK1 (Taq1A) with IGD and its symptoms, and the role of these polymorphisms on IGD and various personality and temperament traits of IGD.

\section{Materials and methods Participants}

We recruited volunteers who agreed with and were able to follow the study design from three online surveys conducted in 2015 and 2016 regarding the Internet gaming behaviors. All participants were assessed using the diagnostic criteria for IGD of the Diagnostic and Statistical Manual of Mental Disorder, 5th edition [31]. The IGD group was defined by endorsement of at least five or more of the nine criteria over a 12-month period. The control subjects were determined as individuals who played the Internet games regularly but were not considered as having IGD. All participants aged 19 or over and had at least 12 years of education.

We interviewed all subjects for the history of neurological or severe medical illness and assessed intellectual function using the Korean version of the Wechsler Adult Intelligence Scale version IV (K-WAIS-IV) to exclude intellectual disability. After excluding four subjects with intellectual disability and one with a history of neurological illness (brain tumor, postoperative status), 63 IGD and 87 control subjects were included in this study. The general characteristics were as follows: age range of 19-47 years old; mean age 30.09 years [standard deviation (SD): 6.343]; number of male participants 115 (76.7\%); time spent gaming on weekdays $2.03 \mathrm{~h}$ (SD: 1.35 ); and time spent gaming on weekends $3.28 \mathrm{~h}$ (SD: 1.94).

The study protocol was approved by Institutional Review Boards of Seoul St. Mary's Hospital (IRB number: KC15EISI0103). This study met the ethical standards of 
the Declaration of Helsinki, including obtaining informed consent from all participants and adhering to the privacy rights of participants.

\section{Measures}

Participants were asked to fill out questionnaires regarding personality and temperament traits as follows: the brief self-control scale (BSCS), the Dickman dysfunctional impulsivity inventory (DII), and the temperament and character inventory-revised short (TCI-RS). Questions about time spent gaming on weekdays and weekends were asked separately.

The BSCS measures the ability to override or change one's inner response as well as to interrupt undesired behavioral tendencies and refrain from acting on them [32]. The BSCS is a 13-item questionnaire with a 5-point Likert scale, and higher scores indicate lower self-control.

The DII is composed of 12 out of 23 items of the Dickman functional and dysfunctional inventory [33]. In DII, dysfunctional impulsivity refers to the tendency to act with less forethought than most people of equal ability when this tendency is a source of difficulty. Items are rated true (1) or false (0) and higher scores indicate higher dysfunctional impulsivity.

The temperament and character inventory (TCI) has been widely used in the investigations of human psychobiological behaviors [34]. TCI measures four temperament dimensions: novelty seeking (NS), harm avoidance (HA), reward dependency (RD), and persistence $(P)$, and three character dimensions: self-directedness (SD), cooperativeness $(C)$, and self-transcendence (ST). We used a shortened TCI-R inventory (TCI-RS), which was consisted of 140 questions [35]. Items on the TCI-RS are rated on a 5-point Likert scale, and higher scores indicate higher prominence of the dimensions.

\section{Genotyping: a specific TaqMan PCR assay}

DNA was isolated from the whole blood using a Wizard Genomic DNA Purification Kit (Promega, Madison, WI, USA) according to the manufacturer's instructions. Genotypes for Taq1A (SNP ID: rs1800497), - 141C Ins/ Del (SNP ID: rs1799732), and C957T (SNP ID: rs6277) polymorphic loci were assessed using the TaqMan SNP genotyping assays (Thermo Fisher Scientific, Carlsbad, CA, USA) on a ViiA 7 Real-Time PCR System (Thermo Fisher Scientific, Foster City, CA, USA). All genotypes were reported using the allelic discrimination program in QuantStudioTM Real-Time PCR Software v1.1 (Thermo Fisher Scientific, Foster City, CA, USA). Only one $-141 C$ genotype of a male control subject was missing. After genotyping, the Taq1A was grouped as the A1+ (homozygous and heterozygous for the A1 allele) and the A1- genotypes (homozygous for the A2 allele), the C957T as the $\mathrm{T}+$ (homozygous and heterozygous for the $\mathrm{T}$ allele) and the $\mathrm{T}$ - genotypes (homozygous for the $\mathrm{C}$ allele), and the $-141 \mathrm{C}$ Ins/Del as the del+ (homozygous and heterozygous for the deletion allele) and del - genotypes (homozygous for the insertion allele).

\section{Statistical analysis}

$X^{2}$ tests were done to investigate the association of polymorphisms with IGD and its symptoms. Independent $t$ tests were carried out to compare the clinical and personality variables between the IGD and control groups except the TCI-RS. Analyses of covariance (ANCOVA) were performed using age as a covariate to explore the TCI-RS profiles between groups since TCI may vary with age [36]. When age had significant interactions with the TCI variables, the results of the independent $t$ tests were present. Subgroup analyses in the IGD group were performed to investigate the associations of the polymorphisms with temperament and personality predispositions in IGD. Binary logistic regression analysis was conducted to calculate the predictive value of each genotype using age and gender as covariates and odds ratio (OR) and $95 \%$ confidential interval (CI) were present. $\chi^{2}$ goodness-of-fit tests were performed in order to calculate the correspondence between the observed number of homozygous and heterozygous individuals and the numbers expected based on Hardy-Weinberg equilibrium. All statistical analyses were performed using SPSS 24.0 (SPSS Inc., Chicago, IL, USA).

\section{Results}

\section{Clinical characteristics and personality traits}

As shown in Table 1, participants with IGD were older (IGD: $32.03 \pm 6.528$, control: $28.68 \pm 5.812, t=-3.398$, $p=.001$ ) and had higher prevalence of male (IGD: 87.4\%, control: $\left.61.9 \%, \chi^{2}=13.232, p=.000\right)$. IGD spent more time on gaming both weekdays and weekends and had higher scores on the BSCS and DII, indicating lower self-control ability and higher dysfunctional impulsivity, than the control subjects. As for the TCIRS, the scores of NS, $P$, and ST were higher in IGD in independent $t$ tests. However, since these three variables had significant interaction effect between group and age, ANCOVA was not performed for them (see Additional file 1 for the scatter plot of ANCOVA and regression slope of NS, $P$, and ST). Other TCI domains showed no significant differences between groups in ANCOVA. Additional subgroup analyses which divided the sample into two groups (age $<30$ vs age $\geq 30$ ) showed no significant differences in TCI-RS domains between groups (results not shown). 
Table 1 Comparisons of clinical and personality variables

\begin{tabular}{lllll}
\hline & Control $(\boldsymbol{n}=\mathbf{8 7})$ & IGD $(\boldsymbol{n}=\mathbf{6 3})$ & $\boldsymbol{x}^{\mathbf{2} / \boldsymbol{t} / \boldsymbol{F}}$ & $\boldsymbol{P}$ \\
\hline Age (years old) & $28.58 \pm 5.812$ & $32.03 \pm 6.528$ & -3.398 & .001 \\
Male proportion (\%) & $76(87.4 \%)$ & $39(61.9 \%)$ & 13.232 & .000 \\
$\begin{array}{l}\text { Weekday gaming } \\
\quad \text { hours }\end{array}$ & $1.66 \pm 1.19$ & $2.57 \pm 1.40$ & -2.805 & .007 \\
$\quad$ Weekend gaming & $2.88 \pm 1.88$ & $3.86 \pm 1.90$ & -2.055 & .044 \\
$\quad$ hours & & & & \\
BSCS & $36.17 \pm 6.547$ & $41.44 \pm 5.482$ & -5.196 & .000 \\
DII & $4.16 \pm 3.429$ & $6.57 \pm 2.988$ & -4.498 & .000 \\
Novelty seeking & $31.59 \pm 8.997$ & $37.54 \pm 8.561$ & $-2.689^{\mathrm{a}}$ & .009 \\
Harm avoidance & $33.104 \pm 2.071$ & $33.666 \pm 1.642$ & .044 & .834 \\
Reward depend- & $42.046 \pm 1.355$ & $44.967 \pm 1.709$ & 1.760 & .189 \\
$\quad$ ence & & & & \\
Persistence & $43.05 \pm 10.538$ & $49.46 \pm 8.659$ & $-2.595^{\mathrm{a}}$ & .012 \\
Self-directedness & $49.368 \pm 1.371$ & $50.727 \pm 1.730$ & .372 & .544 \\
Cooperativeness & $52.759 \pm 1.448$ & $55.534 \pm 1.827$ & 1.391 & .243 \\
Self-transcendence & $18.51 \pm 8.775$ & $24.92 \pm 10.311$ & $-2.725^{\mathrm{a}}$ & .008 \\
\hline
\end{tabular}

IGD Internet gaming disorder, BSCS brief self-control scale, DII Dickman dysfunctional impulsivity scale

${ }^{*} p<.05,{ }^{* *} p<.005$

a Results of $t$ tests

\section{Association of polymorphisms with IGD and its symptoms} Genotype and allele distributions of three polymorphisms were not significantly different between the IGD and control groups (Table 2). The results were similar when analyzed male and female separately. The observed frequencies of homozygous, heterozygous, and non-carriers of the minor alleles were in Hardy-Weinberg equilibrium (Taq1A ANKK1 SNP: $\chi^{2}=0.44, p=.51$; C957T DRD2: $\chi^{2}=0.84, p=.36,-141 C$ DRD2: $\chi^{2}=0.74$, $p=.39$ ).

The association of each genotype with symptoms of IGD was not evident in whole sample (Additional file 2). Subgroup analyses performed in male showed that male with the del-genotype showed higher prevalence of the presence of G6 (Continued excessive use of Internet games despite knowledge of psychosocial problems) and G8 (Use of Internet games to escape or relieve a negative mood) than male with the del+ (Table 3). No association of the genotypes with IGD symptoms was found in female.

\section{Role of ANKK1/DRD2 polymorphisms on IGD}

\section{and personality and temperament predispositions}

In the IGD group, the $t$ tests showed the association of the del + of the $-141 \mathrm{C}$ with higher NS (del+: $44.71 \pm 9.050$, Del-: $34.86 \pm 6.863, t=-2.973, p=.007$ ) (Additional file 3). However, ANCOVA was inapplicable for the investigation of the association of the del genotype and NS due to the significant interaction effect (Additional file 1 ). In subgroup analyses (age $<30$ vs. age $\geq 30$ ), no significant association between genotypes and TCI in the IGD group was found. The Taq1A and C957T polymorphisms were not significantly associated with personality and temperament predispositions in IGD.

Table 4 showed results of the binary logistic regression to predict the risk for IGD using age, gender, and each genotype as covariates. Overall classification accuracy was $72.3 \%$ and Nagelkerke $\mathrm{R}$ square was $23.0 \%$. Being male $(\mathrm{OR}=4.205,95 \%$ CI 1.776-9.956, $p=.001)$ and older age $(\mathrm{OR}=1.098,95 \% \mathrm{CI} 1.034-1.166, p=.002)$ were considered as risk factors for IGD. However, any genotype had no predictive value for being IGD.

\section{Discussion}

The purpose of this preliminary study was to explore the role of the Taq1A ANKK1 and - 141C Ins/Del and C957T DRD2 polymorphisms on IGD. Our results suggested that though these polymorphisms had no association with occurrence and the personality and temperament predispositions in adults with IGD, some symptoms of IGD were associated with the $-141 \mathrm{C}$ Ins/ Del polymorphism in male population.

When compared between the IGD and control groups, IGD subjects had unique clinical and TCI characteristics. First, IGD subjects had higher mean age. The prevalence of IGD across different age groups in adult population has not been investigated before. Given that characteristics of gaming behavior were different across age groups in adults [37], adults with IGD may have unique psychological antecedents that are different from those of adolescents IGD. Second, IGD subjects showed high persistence and self-transcendence as well as low self-control and high impulsivity and novelty seeking as reported before [7, 38-40]. Given that high persistence was associated with perfectionism and obsessive-compulsive disorder $[36,41]$, this may support the compulsive nature of IGD [42]. High self-transcendence may pose the possibility of psychopathology in IGD $[43,44]$. On account of the distribution of allele distribution, our sample was nearly absent in the $\mathrm{T}$ allele of $\mathrm{C} 957 \mathrm{~T}$ and the del of $-141 \mathrm{C}$ DRD2. According to the refSNP database (https://www. ncbi.nlm.nih.gov/projects/SNP/snp_ref.cgi?rs=1799732 and https://www.ncbi.nlm.nih.gov/projects/SNP/snp_ref. cgi?rs $=6277$ ), the prevalence of the $\mathrm{T}$ and del allele was quite low in East Asia, ranging from 0.0487 to 0.0625 and 0.136 , which were comparable to that of our sample, 0.0747 and 0.209 , respectively.

We found that none of the polymorphisms were associated to the occurrence of IGD, which was inconsistent with a previous finding in male adolescents with IGD [7]. Given that adolescents are more eager to take risks and seek for novelties and thus have been considered to be 
Table 2 Allele and genotype frequencies of ANKK1 and DRD2 polymorphisms

\begin{tabular}{|c|c|c|c|c|c|c|c|c|c|}
\hline \multirow[t]{2}{*}{ Genotype } & \multicolumn{3}{|l|}{ ANKK1 Taq 1A } & \multicolumn{3}{|l|}{ DRD2 C952T } & \multicolumn{3}{|c|}{ DRD2 - 141C Ins/Del } \\
\hline & $\mathrm{A} 1 \mathrm{~A} 1$ & $\mathrm{~A} 1 \mathrm{~A} 2$ & $\mathrm{~A} 2 \mathrm{~A} 2$ & TT & $\mathrm{TC}$ & $\mathrm{CC}$ & Del/Del & Del/Ins & Ins/Ins \\
\hline \multicolumn{10}{|l|}{ Whole } \\
\hline Control $(n=87)$ & $15(17.2)$ & $43(48.3)$ & $30(34.5)$ & $0(0.0)$ & $15(17.2)$ & $72(82.8)$ & $1(1.2)$ & $29(33.7)$ & $56(65.1)$ \\
\hline \multirow[t]{2}{*}{$\operatorname{IGD}(n=63)$} & $12(19.0)$ & $26(41.3)$ & 25 (39.7) & $0(0.0)$ & $6(9.5)$ & $57(90.5)$ & $2(3.2)$ & $17(27.0)$ & $44(69.8)$ \\
\hline & \multicolumn{3}{|c|}{$x^{2}=.731, p=.694$} & \multicolumn{3}{|c|}{$x^{2}=1.808, p=.179$} & \multicolumn{3}{|c|}{$x^{2}=1.386, p=.500$} \\
\hline \multicolumn{10}{|l|}{ Male } \\
\hline Control $(n=76)$ & $14(18.4)$ & $35(46.1)$ & $27(35.5)$ & $0(0.0)$ & $12(15.8)$ & $64(84.2)$ & $1(1.3)$ & $26(34.7)$ & $48(64.0)$ \\
\hline \multirow[t]{2}{*}{$\operatorname{IGD}(n=39)$} & $7(17.9)$ & $17(43.6)$ & $15(38.5)$ & $0(0.0)$ & $4(10.3)$ & $35(89.7)$ & $0(0.0)$ & $8(20.5)$ & $31(79.52)$ \\
\hline & \multicolumn{3}{|c|}{$x^{2}=.099, p=.952$} & \multicolumn{3}{|c|}{$x^{2}=.659, p=.417$} & \multicolumn{3}{|c|}{$x^{2}=3.132, p=.209$} \\
\hline \multicolumn{10}{|l|}{ Female } \\
\hline Control $(n=11)$ & \multirow{3}{*}{\multicolumn{3}{|c|}{$\begin{array}{ll}1(9.1) & 7(63.6) \\
5(20.8) & 9(37.5) \\
x^{2}=2.155, p=.341 & \end{array}$}} & \multirow{3}{*}{\multicolumn{3}{|c|}{$\begin{array}{l}0(0.0) \\
0(0.0) \\
x^{2}=2.210, p=.137\end{array}$}} & \multirow{3}{*}{\multicolumn{2}{|c|}{$\begin{array}{ll}0(0.0) & 3(27.3) \\
2(8.3) & 9(37.5) \\
x^{2}=1.580, p=.454 & \\
\end{array}$}} & $8(72.7)$ \\
\hline \multirow{2}{*}{$\operatorname{IGD}(n=24)$} & & & & & & & & & $13(54.2)$ \\
\hline & & & & & & & & & \\
\hline & \multicolumn{3}{|c|}{ ANKK1 Taq 1A } & \multicolumn{3}{|c|}{ DRD2 C952T } & \multicolumn{3}{|c|}{ DRD2 - 141C Ins/Del } \\
\hline Allele & $A 1+$ & & $\mathrm{A} 1-$ & $\mathrm{T}+$ & & $\mathrm{T}-$ & Del+ & & Del- \\
\hline \multicolumn{10}{|l|}{ Whole } \\
\hline Control $(n=87)$ & $57(65.5)$ & & $30(34.1)$ & $15(17.2)$ & & $72(82.8)$ & $30(34.5)$ & & $57(65.5)$ \\
\hline $\operatorname{IGD}(n=63)$ & $38(60.3)$ & & $25(39.7)$ & $6(9.5)$ & & $57(90.5)$ & $19(30.2)$ & & $44(69.8)$ \\
\hline & $x^{2}=.425$ & & & $x^{2}=1.80$ & 179 & & $x^{2}=.368$ & 544 & \\
\hline Male & & & & & & & & & \\
\hline Control $(n=76)$ & $49(64.5)$ & & $27(35.5)$ & $10(16.9)$ & & $49(83.1)$ & $27(36.0)$ & & $48(64.0)$ \\
\hline $\operatorname{IGD}(n=39)$ & $24(61.5)$ & & $15(38.5)$ & $2(5.6)$ & & $34(94.4)$ & $8(20.5)$ & & $31(79.5)$ \\
\hline & $x^{2}=.096$ & & & $x^{2}=.659$ & & & $x^{2}=.2 .89$ & $=.089$ & \\
\hline Female & & & & & & & & & \\
\hline Control $(n=11)$ & $15(51.7)$ & & $14(48.3)$ & $3(27.3)$ & & $8(72.7)$ & $3(27.3)$ & & $8(72.7)$ \\
\hline $\operatorname{IGD}(n=24)$ & $15(55.6)$ & & $12(44.4)$ & $2(8.3)$ & & $22(91.7)$ & $11(45.8)$ & & $13(54.2)$ \\
\hline & $x^{2}=.669$ & 413 & & $x^{2}=2.21$ & .137 & & $x^{2}=1.08$ & .298 & \\
\hline
\end{tabular}

IGD Internet gaming disorder

more susceptible to IGD $[45,46]$, adolescents with risky genetic factors may be more vulnerable to become IGD while adults may be more affected by the complex interaction of genetic and environmental factors. Meanwhile, males with the del-genotype more frequently used Internet games excessively despite knowledge of psychosocial problems and to escape or relieve a negative mood. The del-genotype was associated with alcoholism, especially in male population, and alcoholic patients with the del-continued to drink despite they had protective $A L D H 2 * 2$ and $A D H 1 B * 2$ alleles $[20,47]$. Meanwhile, the role of the $-141 \mathrm{C}$ polymorphism in vivo is controversial; one study showed healthy volunteers with the del+ had higher striatal D2 receptor binding potential while the other showed no significant differences between the del+ and del- $[19,48]$. It can be inferred that the $-141 \mathrm{C}$ $D R D 2$ polymorphism may influence specific behaviors observed in addictive disorders, such as excessive use and compensation of negative mood. Interestingly, this finding was observed only in male subjects. Previous behavioral genetic studies have consistently suggested that alcohol and drug use in males was more determined by genetic factors, while that in female was more by environmental factors $[27,49]$. In addition, male had markedly greater dopamine release than female in the ventral striatum in healthy volunteers, which could account for increased vulnerability of addictive disorders in male [50]. Likewise, gaming behaviors may be more influenced by genetic factors in males than in females due to the neurobiological differences between genders. Further studies are needed to determine different pathways to IGD between male and female.

We found that none of the polymorphisms were related with personality and temperament predispositions in IGD, except the association between the del+ genotype and high novelty seeking. Though age had significant interaction effect, this poses a possibility of the role of the $-141 C$ polymorphism on IGD. In vitro, the 
Table 3 Association of genotypes with symptoms of IGD in male

\begin{tabular}{|c|c|c|c|c|c|c|}
\hline & $A 1+(n=73)$ & $\mathrm{A} 1-(n=42)$ & $T+(n=16)$ & $\mathrm{T}-(n=99)$ & Del+ $(n=35)$ & Del- $(n=79)$ \\
\hline \multirow[t]{2}{*}{ G1 } & $20(27.4 \%)$ & $12(28.6 \%)$ & $4(25.0 \%)$ & $28(28.3 \%)$ & $8(22.9 \%)$ & $24(30.4 \%)$ \\
\hline & $x^{2}=.018, p=.892$ & & $x^{2}=.074, p=.786$ & & $x^{2}=.680, p=.410$ & \\
\hline \multirow[t]{2}{*}{ G2 } & $16(21.9 \%)$ & $12(28.6 \%)$ & $3(18.8 \%)$ & $25(25.3 \%)$ & $6(21.4 \%)$ & $22(27.8 \%)$ \\
\hline & $x^{2}=.641, p=.423$ & & $x^{2}=.316, p=.574$ & & $x^{2}=1.500, p=.221$ & \\
\hline \multirow[t]{2}{*}{ G3 } & $22(30.1 \%)$ & $14(33.3 \%)$ & $3(18.8 \%)$ & 33 (33.3\%) & $8(22.9 \%)$ & $28(35.4 \%)$ \\
\hline & $x^{2}=.127, p=.722$ & & $x^{2}=1.362, p=.243$ & & $x^{2}=1.778, p=.182$ & \\
\hline \multirow[t]{2}{*}{ G4 } & $27(37.0 \%)$ & $18(42.9 \%)$ & $7(43.8 \%)$ & 38 (37.4\%) & $11(31.4 \%)$ & $34(43.0 \%)$ \\
\hline & $x^{2}=.386, p=.535$ & & $x^{2}=.167, p=.683$ & & $x^{2}=1.368, p=.242$ & \\
\hline \multirow[t]{2}{*}{ G5 } & $24(32.9 \%)$ & 19 (45.2\%) & $7(43.8 \%)$ & $36(36.4 \%)$ & $10(28.6 \%)$ & $33(41.8 \%)$ \\
\hline & $x^{2}=1.740, p=.187$ & & $x^{2}=.321, p=.571$ & & $x^{2}=1.779, p=.180$ & \\
\hline \multirow[t]{2}{*}{ G6 } & $21(28.8 \%)$ & $13(31.0 \%)$ & $5(31.3 \%)$ & $29(29.3 \%)$ & $6(17.1 \%)$ & $28(35.4 \%)$ \\
\hline & $x^{2}=.061, p=.805$ & & $x^{2}=.025, p=.874$ & & $x^{2}=3.881, p=.049^{*}$ & \\
\hline \multirow[t]{2}{*}{ G7 } & $27(37.0 \%)$ & 18 (42.9\%) & $5(31.3 \%)$ & $40(40.4 \%)$ & $11(31.4 \%)$ & $34(43.0 \%)$ \\
\hline & $x^{2}=.386, p=.535$ & & $x^{2}=.485, p=.486$ & & $x^{2}=.368, p=.242$ & \\
\hline \multirow[t]{2}{*}{ G8 } & $20(27.4 \%)$ & 15 (35.7\%) & $5(31.3 \%)$ & $30(30.3 \%)$ & $6(17.1 \%)$ & $29(36.7 \%)$ \\
\hline & $x^{2}=.871, p=.351$ & & $x^{2}=.006, p=.939$ & & $x^{2}=4.364, p=.037^{*}$ & \\
\hline \multirow[t]{2}{*}{ G9 } & $14(19.2 \%)$ & $10(23.8 \%)$ & $2(12.5 \%)$ & $22(22.2 \%)$ & $4(11.4 \%)$ & $20(25.3 \%)$ \\
\hline & $x^{2}=.346, p=.556$ & & $x^{2}=.788, p=.375$ & & $x^{2}=2.815, p=.093$ & \\
\hline
\end{tabular}

G1-G9 represented DSM-5 IGD criteria (Additional file 2)

${ }^{*} p<.05$

Table 4 Predictive values for Internet gaming disorder

\begin{tabular}{lrrrrl}
\hline Variables & \multicolumn{1}{c}{$\boldsymbol{B}$} & S.E. & OR & $\mathbf{9 5 \% \mathrm { Cl }}$ & $\boldsymbol{p}$ \\
\hline Male gender & 1.436 & .440 & 4.205 & $1.776-9.956$ & $.001^{* *}$ \\
Age & .094 & .031 & 1.098 & $1.034-1.166$ & $.002^{* *}$ \\
A1+ of Taq1A ANKK1 & -.534 & .399 & .586 & $.268-1.281$ & .180 \\
T+ of C957T DRD2 & -1.161 & .607 & .559 & $.255-1.225$ & .146 \\
Del+ of - 141C DRD2 & -2.797 & .950 & .313 & $.095-1.028$ & .056 \\
\hline
\end{tabular}

$\mathrm{Cl}$ confidential interval

${ }^{* *} p<.005,{ }^{*} p<.05$

del allele-containing construct showed marked reduction in promoter activity, manifesting only $21-43 \%$ of the reporter gene expression level attributed to the ins allelecontaining construct [16]. In addition, the del+ genotype showed differential neural response when performing the Go/No-go task in alcohol-abusing adults [51], suggesting the role of $-141 \mathrm{C}$ polymorphism in important neurocognitive function. A recent study on the heritability of Internet addiction (IA) suggested that genetic factors accounted for $20-65 \%$ of a part of variance of specific IA factors such as personality factor self-directedness; they had negligible influence on generalized facets of IA [52]. Likewise, genetic factors may account for a certain part of the variance of temperament and personality traits observed in IGD. Though the del-genotype itself has been associated with alcohol dependence $[20,47,53]$, the
- 141C polymorphism may have different roles in IGD possibly by mediating temperament traits.

Neither univariate and multivariate logistic regression analyses showed predictive value of polymorphisms of interest for the occurrence of IGD in adult population. Though we could not find the direct association of these polymorphisms with IGD, it is too hasty to draw final conclusion that these polymorphisms have no role in IGD. Given that genes interact with other genes as well as environmental factors and modify gene or protein expression levels even not altering DNA sequences, further researches using high-throughput technologies would give opportunities to explore these epistatic or epigenetic changes made in IGD.

This study had several limitations that should be noted. First, the sample size was relatively small given the possibility of false-positive findings in studies with small samples. However, this is a preliminary study to explore the role of the DRD2 and $A N K K 1$ polymorphisms on IGD and would give an insight to the theme. Second, the IGD and control group had different mean age and gender distribution, which may confound the results. However, subgroup analyses in both genders and different age groups (age $<30$ vs. age $\geq 30$ ) showed comparable results with the original analyses. Third, psychiatric comorbidities such as depression or anxiety disorder and substance addictions were not screened. Nevertheless, since our sample was composed of non-clinical population and 
subjects with comparable intelligence levels, the proportion of psychiatric comorbidities is supposed to be small.

Despite the limitations, this study had some methodological strengths. First, our study focused on the adult population, which was a remarkable departure from previous studies that mainly focused on male adolescents. Since adolescents are susceptible to addictive disorder [46], the investigation of genetic predispositions in adult population would give more accurate perspectives by regressing out the contribution of characteristics of adolescents in the development of IGD. Second, we compared the IGD subjects with casual gamers. Considering that only a small portion of gamers become IGD out of casual gamers, this method had an advantage to investigate the neurobiological mechanisms that differentiate IGD from casual gamers. Third, the sample was homogeneous with respect to the ethnicity, education, and intellectual levels. By using interviews and objective measures, we excluded participants with neurological disorders and intellectual disabilities which may severely affect the personality and temperament traits.

\section{Conclusions}

Our findings suggest that the genetic variations of the - $141 C$ DRD2 may play a role on IGD via mediating specific symptoms or temperament traits in adult population, especially in male gender, though no direct association was observed. The neurobiological mechanisms of IGD may be different between adults and adolescents and between male and female. Further researches are necessary to investigate the interaction between genes and environmental factors and the differences across genders and age groups in neurobiological and psychological antecedents of IGD.

\section{Additional files}

Additional file 1. Scatter plot with regression line of results of ANCOVA.

Additional file 2. Genotype and allele frequency for each criteria of DSM-5 IGD.

Additional file 3. Comparisons of personality and temperament between genotypes in Internet gaming disorder.

\section{Authors' contributions}

All the authors contributed to the design of the study. S-HP, MRC, J-WC, $\mathrm{HJ}, \mathrm{J}-\mathrm{EJ}$, and D-JK contributed to study planning and advised to the course of study. J-WC, J-YK, J-HC, HJ, and J-EJ conducted the data collection from participants. S-HP conducted the literature search and provided summaries of previous research studies. MRC, SMK, and SHB conducted the genotyping. S-HP and MRC conducted the statistical analysis and wrote the first draft of the manuscript. All authors contributed to the development of the manuscript and revised it critically. All authors read and approved the final manuscript.

Acknowledgements

Not applicable.

\section{Competing interests}

The authors declare that they have no competing interests. The authors alone are responsible for the content and writing of this paper.

\section{Availability of data and supporting materials}

The data will not be shared.

\section{Ethics approval and consent to participate}

The study protocol was approved by Institutional Review Boards of Seoul St. Mary's Hospital (IRB Number: KC15EISI0103). This study met the ethical standards of the Declaration of Helsinki, including obtaining informed consent from all participants and adhering to the privacy rights of participants.

\section{Funding}

This research was supported by a grant from the Brain Research Program through the National Research Foundation of Korea (NRF) funded by the Ministry of Science, ICT, \& Future Planning (NRF-2014M3C7A1062893). The funding source had no role in the study design, collection, analysis or interpretation of data, writing of the manuscript, or the decision to submit the paper for publication.

\section{Publisher's Note}

Springer Nature remains neutral with regard to jurisdictional claims in published maps and institutional affiliations.

Received: 17 May 2017 Accepted: 28 November 2017

Published online: 08 December 2017

References

1. American Psychiatry Association. Diagnostic and statistical manual of mental disorders. 5th ed. Arlington, VA: American Psychiatric Association; 2013.

2. Grant JE, Potenza MN, Weinstein A, Gorelick DA. Introduction to behavioral addictions. Am J Drug Alcohol Abuse. 2010;36(5):233-41.

3. Zhou Z, Yuan G, Yao J. Cognitive biases toward Internet game-related pictures and executive deficits in individuals with an Internet game addiction. PLoS ONE. 2012;7(11):e48961.

4. Yao Y-W, Chen P-R, Li S, Wang L-J, Zhang J-T, Yip SW, Chen G, Deng L-Y, Liu Q-X, Fang X-Y. Decision-making for risky gains and losses among college students with Internet gaming disorder. PLoS ONE. 2015;10(1):e0116471.

5. Yao Y-W, Wang L-J, Yip SW, Chen P-R, Li S, Xu J, Zhang J-T, Deng L-Y, Liu Q-X, Fang X-Y. Impaired decision-making under risk is associated with gaming-specific inhibition deficits among college students with Internet gaming disorder. Psychiatr Res. 2015;229(1):302-9.

6. Tian M, Chen Q, Zhang Y, Du F, Hou H, Chao F, Zhang H. PET imaging reveals brain functional changes in Internet gaming disorder. Eur J Nucl Med Mol Imaging. 2014;41(7):1388-97.

7. Han DH, Lee YS, Yang KC, Kim EY, Lyoo IK, Renshaw PF. Dopamine genes and reward dependence in adolescents with excessive Internet video game play. J Addict Med. 2007;1 (3):133-8.

8. Li M, Chen J, Li N, Li X. A twin study of problematic Internet use: its heritability and genetic association with effortful control. Twin Res Hum Genet. 2014;17(4):279-87.

9. Long EC, Verhulst B, Neale MC, Lind PA, Hickie IB, Martin NG, Gillespie NA. The genetic and environmental contributions to Internet use and associations with psychopathology: a twin study. Twin Res Hum Genet. 2016;19(1):1-9.

10. Vink JM, Beijsterveldt TC, Huppertz C, Bartels M, Boomsma DI. Heritability of compulsive Internet use in adolescents. Addict Biol. 2016;21(2):460-8.

11. Kreek MJ, Nielsen DA, Butelman ER, LaForge KS. Genetic influences on impulsivity, risk taking, stress responsivity and vulnerability to drug abuse and addiction. Nat Neurosci. 2005;8(11):1450.

12. Comings DE, Blum K. Reward deficiency syndrome: genetic aspects of behavioral disorders. Prog Brain Res. 2000;126:325-41.

13. Noble EP. The DRD2 gene in psychiatric and neurological disorders and its phenotypes. Pharmacogenomics. 2000;1(3):309-33. 
14. Doehring A, Kirchhof A, Lötsch J. Genetic diagnostics of functional variants of the human dopamine D2 receptor gene. Psychiatr Genet. 2009;19(5):259-68.

15. Neville MJ, Johnstone EC, Walton RT. Identification and characterization of ANKK1: a novel kinase gene closely linked to DRD2 on chromosome band 11q23. 1. Hum Mutat. 2004;23(6):540-5.

16. Arinami T, Gao M, Hamaguchi $H$, Toru $M$. A functional polymorphism in the promoter region of the dopamine $\mathrm{D} 2$ receptor gene is associated with schizophrenia. Hum Mol Genet. 1997;6(4):577-82.

17. Seeman $P$, Ohara K, Ulpian C, Seeman MV, Jellinger $K$, Van Tol HH, Niznik HB. Schizophrenia: normal sequence in the dopamine D2 receptor region that couples to G-proteins. DNA polymorphisms in D2. Neuropsychopharmacology. 1993;8(2):137-42.

18. Ponce G, Hoenicka J, Jimenez-Arriero M, Rodriguez-Jimenez R, Aragüés M, Martin-Sune N, Huertas E, Palomo T. DRD2 and ANKK1 genotype in alcohol-dependent patients with psychopathic traits: association and interaction study. Br J Psychiatr. 2008;193(2):121-5.

19. Jönsson E, Nöthen $M$, Grünhage F, Farde L, Nakashima Y, Propping P, Sedvall G. Polymorphisms in the dopamine D2 receptor gene and their relationships to striatal dopamine receptor density of healthy volunteers. Mol Psychiatry. 1999;4(3):290-6.

20. Konishi T, Luo HR, Calvillo M, Mayo MS, Lin KM, Wan YJY. ADH1B* 1 , $\mathrm{ADH}_{1} \mathrm{C}^{*}$ 2, DRD2 (-141C Ins), and 5-HTTLPR are associated with alcoholism in Mexican American men living in Los Angeles. Alcohol Clin Exp Res. 2004;28(8):1145-52.

21. Montag C, Flierl M, Markett S, Walter N, Jurkiewicz M, Reuter M. Internet addiction and personality in first-person-shooter video gamers. Media Psychol. 2011;23:163-73.

22. Montag C, Jurkiewicz M, Reuter M. Low self-directedness is a better predictor for problematic Internet use than high neuroticism. Comput Human Behav. 2010;26(6):1531-5.

23. Ha JH, Kim SY, Bae SC, Bae S, Kim H, Sim M, Lyoo IK, Cho SC. Depression and Internet addiction in adolescents. Psychopathology. 2007:40(6):424-30.

24. Cao F, Su L. Internet addiction among Chinese adolescents: prevalence and psychological features. Child Care Health Dev. 2007;33(3):275-81.

25. Jang KL, Livesley WJ, Vemon PA. Heritability of the big five personality dimensions and their facets: a twin study. J Pers. 1996;64(3):577-92.

26. Polderman TJ, Benyamin B, De Leeuw CA, Sullivan PF, Van Bochoven A, Visscher PM, Posthuma D. Meta-analysis of the heritability of human traits based on 50 years of twin studies. Nat Genet. 2015;47(7):702-9.

27. Munafo MR, Clark TG, Moore LR, Payne E, Walton R, Flint J. Genetic polymorphisms and personality in healthy adults: a systematic review and meta-analysis. Mol Psychiatry. 2003;8(5):471.

28. Gebhardt C, Leisch F, Schüssler P, Fuchs K, Stompe T, Sieghart W, Hornik $\mathrm{K}$, Kasper S, Aschauer H. Non-association of dopamine D4 and D2 receptor genes with personality in healthy individuals. Psychiatr Genet. 2000;10(3):131-7.

29. Kwon YS, Lim S, Shin YC. An association study of the dopamine D2 receptor Taq1A polymorphism and temperament in Korean pathological gamblers. Korean J Biol Psychiatry. 2011;18(3):119-25.

30. Lee YS, Han DH, Yang KC, Daniels MA, Na C, Kee BS, Renshaw PF. Depression like characteristics of 5 HTTLPR polymorphism and temperament in excessive Internet users. J Affect Disord. 2008;109(1):165-9.

31. Pontes HM, Kiraly O, Demetrovics Z, Griffiths MD. The conceptualisation and measurement of DSM-5 Internet gaming disorder: the development of the IGD-20 test. PLoS ONE. 2014;9(10):e110137.

32. Tangney JP, Baumeister RF, Boone AL. High self-control predicts good adjustment, less pathology, better grades, and interpersonal success. J Pers. 2004;72(2):271-324.

33. Dickman SJ. Functional and dysfunctional impulsivity: personality and cognitive correlates. J Pers Soc Psychol. 1990;58(1):95.

34. Cloninger CR, Svrakic DM, Przybeck TR. A psychobiological model of temperament and character. Arch Gen Psychiatry. 1993;50(12):975-90.

35. Farmer RF, Goldberg LR. A psychometric evaluation of the revised temperament and Character Inventory (TCI-R) and the TCl-140. Psychol Assess. 2008;20(3):281
36. Josefsson K, Jokela M, Cloninger CR, Hintsanen M, Salo J, Hintsa T, PulkkiRåback L, Keltikangas-Järvinen L. Maturity and change in personality: developmental trends of temperament and character in adulthood. Dev Psychopathol. 2013;25(3):713-27.

37. Korea Creative Content Agency (KCCA): a survey on game usage 2016. http://portal.kocca.kr/cop/bbs/view/B0000147/1831102. do?menuNo=200904. Accessed Feb 22, 2017

38. Lin SS, Tsai C-C. Sensation seeking and Internet dependence of Taiwanese high school adolescents. Comput Hum Behav. 2002;18(4):411-26.

39. Dalbudak E, Evren C, Aldemir S, Coskun KS, Ugurlu H, Yildirim FG. Relationship of Internet addiction severity with depression, anxiety, and alexithymia, temperament and character in university students. Cyberpsychol Behav Soc Netw. 2013;16(4):272-8.

40. Kim EJ, Namkoong K, Ku T, Kim SJ. The relationship between online game addiction and aggression, self-control and narcissistic personality traits. Eur Psychiatry. 2008;23(3):212-8.

41. Mulder RT, Joyce PR. Temperament and the structure of personality disorder symptoms. Psychol Med. 1997;27(1):99-106.

42. Van Rooij AJ, Schoenmakers TM, Van de Eijnden RJ, Van de Mheen D. Compulsive Internet use: the role of online gaming and other Internet applications. J Adol Health. 2010;47(1):51-7.

43. Cloninger $\mathrm{CR}$, Zohar $\mathrm{AH}$. Personality and the perception of health and happiness. J Affect Disord. 2011;128(1):24-32.

44. Cloninger $\mathrm{CR}$, Zohar $\mathrm{AH}$, Cloninger $\mathrm{KM}$. Promotion of well-being in person-centered mental health care. Focus. 2010;8(2):165-79.

45. Griffiths M, Wood RT. Risk factors in adolescence: the case of gambling, videogame playing, and the Internet. J Gambl Stud. 2000;16(2):199-225.

46. Crews F, He J, Hodge C. Adolescent cortical development: a critical period of vulnerability for addiction. Pharmacol Biochem Behav. 2007:86(2):189-99.

47. Ishiguro $\mathrm{H}$, Arinami T, Saito T, Akazawa S, Enomoto M, Mitushio H, Fujishiro H, Tada K, Akimoto Y, Mifune H. Association study between the 441 c ins/del and taqi a polymorphisms of the dopamine $\mathrm{d} 2$ receptor gene and alcoholism. Alcohol Clin Exp Res. 1998;22(4):845-8.

48. Pohjalainen T, Någren K, Syvälahti E, Hietala J. The dopamine D2 receptor 5'-flanking variant, $-141 \mathrm{C}$ Ins/Del, is not associated with reduced dopamine D2 receptor density in vivo. Pharmacogenet Genom. 1999;9(4):505-9.

49. Jang KL, Livesley W, Vernon PA. Gender-specific etiological differences in alcohol and drug problems: a behavioural genetic analysis. Addiction. 1997;92(10):1265-76.

50. Munro CA, McCaul ME, Wong DF, Oswald LM, Zhou Y, Brasic J, Kuwabara $\mathrm{H}$, Kumar A, Alexander M, Ye W. Sex differences in striatal dopamine release in healthy adults. Biol Psychiatry. 2006;59(10):966-74.

51. Filbey FM, Claus ED, Morgan M, Forester GR, Hutchison K. Dopaminergic genes modulate response inhibition in alcohol abusing adults. Addict Biol. 2012;17(6):1046-56.

52. Hahn E, Reuter M, Spinath FM, Montag C. Internet addiction and its facets: the role of genetics and the relation to self-directedness. Addict Behav. 2017;65:137-46.

53. Prasad P, Ambekar A, Vaswani M. Dopamine D2 receptor polymorphisms and susceptibility to alcohol dependence in Indian males: a preliminary study. BMC Med Genet. 2010;11(1):24.

\section{Submit your next manuscript to BioMed Central and we will help you at every step:}

- We accept pre-submission inquiries

- Our selector tool helps you to find the most relevant journal

- We provide round the clock customer support

- Convenient online submission

- Thorough peer review

- Inclusion in PubMed and all major indexing services

- Maximum visibility for your research

Submit your manuscript at www.biomedcentral.com/submit 